This article was published in Materials Research Bulletin, 61, 528-533, 2015

http://dx.doi.org/10.1016/j.materresbull.2014.10.055

\title{
Deposition of Pd-Ag thin film membranes on ceramic supports for hydrogen purification/separation
}

\author{
A.I. Pereira a , P. Pérez b, S.C. Rodrigues b, A. Mendes b, L.M. Madeira b, C.J. \\ Tavares ${ }^{a, *}$ \\ a Centre of Physics, University of Minho, Campus Azurém, 4800-058, Portugal \\ b LEPAE, Chemical Engineering Department, Faculty of Engineering, University of \\ Porto, Rua Dr. Roberto Frias s/n, 4200-465 Porto, Portugal
}

\begin{abstract}
$\mathrm{Pd}-\mathrm{Ag}$ based membranes supported on porous a-Al2O3 (doped with yttriastabilized zirconia) were studied for hydrogen selective separation. Magnetron sputtering technique was employed for the synthesis of thin film membranes. The hydrogen permeation flux is affected by the membrane columnar structure, which is formed during deposition. From scanning electron microscopy analysis, it was observed that different sputtering deposition pressures lead to distinct columnar structure growth. X-ray diffraction patterns provided evidence of a Pd-Ag solid solution with an average crystallite domain size of $21 \mathrm{~nm}$, whose preferential growth can be altered by the deposition pressure. The gaspermeation results have shown that the $\mathrm{Pd}-\mathrm{Ag}$ membrane supported on porous $a-\mathrm{Al} 2 \mathrm{O} 3$ is selective toward $\mathrm{H} 2$. For optimized membrane synthesis conditions, the permeance toward N2 is $0.076 \times 10^{-6} \mathrm{~mol} \mathrm{~m}^{-2} \mathrm{~s}^{-1} \mathrm{~Pa}^{-1}$ at room temperature, whereas for a pressure difference of $300 \mathrm{kPa}$ the $\mathrm{H} 2-$ flux is of the order of ca. $0.21 \mathrm{~mol} \mathrm{~m}^{-2} \mathrm{~s}^{-1}$, which corresponds to a permeance of $0.71 \times 10^{-6} \mathrm{~mol} \mathrm{~m}^{-2} \mathrm{~s}^{-1} \mathrm{~Pa}^{-1}$, yielding a selectivity of $a$ $(\mathrm{H} 2 / \mathrm{N} 2)=10$. These findings suggest that the membrane has a reasonable capacity to selectively permeate this gas.
\end{abstract}

\section{Introduction}

Palladium (Pd)-based membranes have been studied for many years, regarding applications in production and purification of hydrogen. The reaction of the water gas shift $\left(\mathrm{CO}+\mathrm{H} 2 \mathrm{O} \leftrightarrow \mathrm{CO}_{2}+\mathrm{H}_{2}\right)$, for example, can advantageously be conducted in a Pd-based membrane reactor, where hydrogen selectively permeates the membrane [1]. Another example where these membranes find the application is in the largest industrial process for $\mathrm{H}_{2}$ production: the methane steam reforming 
$\left(\mathrm{CH} 4+\mathrm{H}_{2} \mathrm{O} ? \mathrm{CO}+3 \mathrm{H} 2\right)$ [2]. When hydrogen permeates with an infinite selectivity, its passage is governed by sorption-diffusion mechanism through the atomic structure. Molecular hydrogen adsorbs on the membrane surface, dissociates into atomic hydrogen, diffuses in the atomic form through the metal bulk and recombines into hydrogen molecules at the other membrane surface [3].

Among all metals, palladium is the material that exhibits the highest atomic hydrogen permeability, resulting from the high capability in the catalytic dissociation of $\mathrm{H} 2$ molecules in its surface, following by dissolution in the metal structure [4]. However, the use of pure palladium membranes has some limitations. The hydrogen concentration increases during the permeation below its critical point of $571 \mathrm{~K}$ and $2 \mathrm{MPa}$, producing two hybrid phases ( $a$ and $b$ ) [5]. Both have a face-centered cubic (fcc) structure, but different cell lattice parameters, leading to an increase of tension in the membrane [4]. With the increase of tension, palladium loses ductility due to the exposure to hydrogen, causing cracking in the metal, which is commonly known as hydrogen embrittlement $[4,6]$. Furthermore, besides the fact that the raw Pd metal has a high cost, in corrosive environments these membranes may be prone to irreversible poisoning by sulfur, carbon monoxide and carbon dioxide [6].

When palladium alloys such as Pd-Ag is used, the result is a homogeneous solid solution with an fcc structure [7,8]. This alloy prevents the formation of particular hybrid phases, allowing for higher hydrogen permeation along with chemical and mechanical stability, reducing also the overall cost of raw material [4].

Structurally, membranes are classified in two groups: composite and selfsupported. In order to obtain thin metal films, porous supports are used to increase mechanical strength and thermal stability $[4,9,10]$, being the most commonly used made of porous stainless steel, ceramics and glass [9]. The mechanism of mass transport is now predominantly of the Knudsen type due to the existence of small pores, however dependent on specific conditions of the reactor, thickness and pore size of the individual layers of the heterostructured membrane. However, a porous support inevitably offers a higher superficial roughness and hosts imperfections due to porosity, leading to the formation of pinholes and imperfections in the thin film membrane [4].

Alumina (a-Al2O3) has been reported as one of the most used membrane supports. Upon preparation of the composite porous support, the introduction of thin layers of $g-\mathrm{Al} 2 \mathrm{O} 3$ by dip coating affects the hydrogen permeation flux and selectivity. This modification results in a pinhole-free and stable membrane $[11,12]$. Recently, Tanaka et al. reported the doping of $g$-Al2O3 with yttriastabilized zirconia (YSZ), prepared by dip coating [13]. This thin layer deposited on the alumina support enhances the adhesion and uniformity of the thin film membrane. Furthermore, the thermal expansion coefficient of zirconia is in the range between that of palladium and alumina; hence, it is tailored to yield an 
excellent flexural strength, fracture toughness and fatigue resistance [13]. In addition, the pore dimension of the membrane support is reduced (e.g., from 200 to ca. $5 \mathrm{~nm}$ ), facilitating the deposition of the Pd-Ag film.

The main techniques used for producing Pd-based membranes are electroless plating, chemical vapor deposition [14], sol-gel [15], cold rolling, magnetron sputtering and electroplating deposition [16]. In order to have a feasible and economic potential, any of these techniques should ensure that the membrane have a high hydrogen permeability, good selectivity over other gases and is able to keep its performance for a long time under elevated temperatures and feed pressures [4]. The synthesis of membranes by magnetron sputtering, with optimized processing parameters, originates a thin film with good quality, mostly free of voids and pinholes, with a high coverage on the substrate in order to prevent any leakage $[16,17]$. Furthermore, it has the important advantage of allowing the production of very thin films, of the order of hundreds of nanometers, decreasing the membrane cost while increasing hydrogen permeation flux. However, if the support has initially a very high roughness the coating will not ensure a uniform deposition over the support, leading to different microstructural morphologies in the membrane that hinder densification of the microstructure $[18,19]$.

In this study, $\mathrm{Pd}-\mathrm{Ag}$ based membranes were produced on alumina porous supports (previously doped with yttria-stabilized zirconia) by magnetron sputtering technique. The deposition parameters were optimized concerning the membrane selectivity toward $\mathrm{H}_{2} / \mathrm{N} 2$. Permeation tests were carried out to assess the selectivity and hydrogen permeation of the membrane. The membrane cross-section microstructure, surface morphology and thickness were analyzed by scanning electron microscopy (SEM). By coupling SEM with energy dispersive X-ray spectroscopy (EDX) it enabled the chemical assessment of the thin film composition, which is very important for membrane quality control and reproducibility at a later stage of a tentative industrial scale-up. X-ray diffraction (XRD) was used to characterize the membrane crystallinity.

\section{Materials and methods}

A porous $\alpha-\mathrm{Al} 2 \mathrm{O} 3(99.9 \%)$ tube with an average pore size of $200 \mathrm{~nm}$ was used as membrane support. The $\gamma-\mathrm{Al}_{2} \mathrm{O} 3$ with yttria-stabilized zirconia layer (YSZ- $\gamma-\mathrm{Al} 2 \mathrm{O} 3$ ) was produced by a sol-gel technique by dip-coating onto the $a$ $\mathrm{Al} 2 \mathrm{O} 3$ porous support, then subsequently dried at $40^{\circ} \mathrm{C}$ and calcinated at $550{ }^{\circ} \mathrm{C}$, as described in detail elsewhere [13]. This layer was added to create a smoother surface that allows an enhanced adhesion of the $\mathrm{Pd}-\mathrm{Ag}$ film membrane.

Palladium and silver were deposited onto the $\mathrm{YSZ}-\gamma-\mathrm{Al} 2 \mathrm{O} 3$ layer by magnetron 
sputtering (MS). MS is a type of physical vapor deposition (PVD) technique, using high vacuum and metal target- loaded magnetrons. This method consists in bombarding both Pd and Ag targets with energetic argon ions and subsequent ejection of the neutral metal atom species, which will then condense as a growing thin film on the support. Fig. 1 shows a sketch that illustrates the co-sputtering process from two magnetrons in the customized PVD equipment. The process parameters were: base pressure $\approx 10^{-4} \mathrm{~Pa}$; working pressure $=0.37-3 \mathrm{~Pa}$; target current density $(\mathrm{Pd})=7.6-10 \mathrm{~mA} / \mathrm{cm}^{2}$; target current density $(\mathrm{Ag})=1.6 \mathrm{~mA} / \mathrm{cm}^{2}$; bias voltage applied to support holder $=-60 \mathrm{~V}$, working gas flow $(\mathrm{Ar})=35-50$ sccm. Table 1 displays more details for each sample. Prior to deposition the supports were etched in argon plasma at $1 \mathrm{~Pa}$ for a maximum of $15 \mathrm{~min}$. During deposition, the support was heated to $\sim 200^{\circ} \mathrm{C}$ and rotated vertically with a speed of $19 \mathrm{rpm}$ between both inclined magnetrons, as it can be seen in Fig. 1.

Gas-permeation experiments were carried out to determine the permeation to nitrogen and to hydrogen. First, the membranes were tested only with nitrogen to evaluate the ability to reject other gases than hydrogen. If the permeance toward nitrogen would prove to be low, which in our case was set to a lower limit of $0.1 \times 10^{-6} \mathrm{~mol} \mathrm{~m}^{-2} \mathrm{~s}^{-1} \mathrm{~Pa}^{-1}$ at room temperature, the experimental procedure proceeded to the determination of the hydrogen/ nitrogen ideal selectivity. The permeation tests were performed at room temperature (RT) and flow rates converted toSTP conditions; a schematic of this experimental set-up is given in Fig. 2. The pressure difference, $\Delta P=(P$ feed $P$ permeate $)$, through the membrane varied between 100 and $500 \mathrm{kPa}$, and the permeate pressure was set to the ambient pressure. The permeate flow rate was measured using a bubble meter Horiba STEC VP-3. The reactor that was used for the gas-permeation experiments (Fig. 2) can contribute to some limitations in the assessment of the permeability of the membranes. First, it is important to seal all connections between the gas lines and the testing non-porous ceramic support. Prior to the experiments, these seals were thoroughly checked with a bubble meter. Furthermore, exceptional care must be taken in the glass seal between the nonporous and active porous support onto which the thin film will be deposited. Failure in this will result in a high permeation through these leaks, preventing proper analysis.

SEM and EDX were used to observe the surface and cross section of the PVDdeposited Pd-Ag thin film membranes, and $\mathrm{YSZ}-\gamma-\mathrm{Al} 2 \mathrm{O} 3$ interface layer, supported on the $\alpha-\mathrm{Al} 2 \mathrm{O} 3$ tube. A FEI Nova 200 (FEG/SEM) apparatus coupled with EDAX Pegasus-X4 M (EDX) was used for this purpose. For X-ray diffraction experiments, a Bruker AXS D8 Discover diffractometer with $\mathrm{Cu} K a$ radiation in the $\theta-2 \theta$ geometry was used to analyze the crystalline structure. Bruker AXS TOPAS software was used for the Rietveld refinement of the XRD data. 


\section{Results and discussion}

Fig. 3 illustrates the porous $a-\mathrm{Al} 2 \mathrm{O} 3$ support pretreated with the sol-gel YSZ$\gamma$-Al2O3 layer (top) and after $\mathrm{Pd}-\mathrm{Ag}$ thin film deposition (bottom). The morphology of the PVD-deposited Pd-Ag thin films can be observed in the SEM micrographs of Fig. 4, taken from a series of sample membranes A1, A2, A3 (see Table 1). In this series, the process parameters remained constant, except for the sputtering pressure. Albeit the sputtering gas (argon) flow rate was kept constant at $50 \mathrm{sccm}$, resulting in a sputtering pressure of $0.43 \mathrm{~Pa}$ for the deposition of sample A1, for samples A2 and A3 the pressure was increased by adjusting the throttle valve of the pumping system. Thus, from the SEM micrographs in Fig. 4 , it is obvious that upon increasing the sputtering pressure from $0.43 \mathrm{~Pa}$ to $3 \mathrm{~Pa}$ the morphology resulting from the top of the columnar structure becomes rougher: this is more evident for membrane A3, with wider grains surrounded by larger voids, in contrast to the more dense structure seen for membrane A1. Membrane A1 has a morphology characteristic of zone T in Thornton's model, while the A3 clearly resembles zone 1 [19].

From this series of samples, gas-permeation measurements were performed to determine the permeance of membrane A1 to nitrogen, which hosts a more compact structure. A very high permeance of $0.91 \times 10^{-6} \mathrm{~mol} \mathrm{~m}^{-2} \mathrm{~s}^{-1} \mathrm{~Pa}^{-1}$ was registered for this membrane, as reported in Table 1. Close inspection of its microstructure on several points of its surface revealed tensile cracks resulting from excessive residual stress in the Pd-Ag thin film; these cracks can be seen on the top part of the micrograph corresponding to this membrane (indicated by arrows in Fig. 4a). As the deposition pressure increased from 0.43 to $3 \mathrm{~Pa}$ in this series of membranes, the measured silver content in the $\mathrm{Pd}-\mathrm{Ag}$ film also increased, due to the concomitant increase in silver sputtering yield. One would expect a decrease in the film thickness with this variation in pressure. However, it can be seen in Table 1 that in fact the sputtering yield increase escalates the film thickness, counter- acting the reduction in mean free path of the sputtered atoms and reduced adatom mobility on the growing film surface. Thus, from observing Fig. 4, it should be expected for membrane A1 an optimized microstructure for hydrogen selectivity, notwithstanding the need to decrease internal stresses in the film.

Fig. 5 shows the XRD patterns of membrane samples A1 and A3, deposited with the minimum (0.43 Pa) and maximum (3 Pa) sputtering pressure. The average crystalline domain size increases slightly from $20 \mathrm{~nm}$ (A1) to $22 \mathrm{~nm}$ (A3) with growing deposition pressure; this change is however within the size determination error $( \pm 2 \mathrm{~nm})$. Nevertheless, it is observed that the stress state and crystalline growth direction changes from sample A1 to sample A3. While sample A1 shows a prominent reflection associated with a face-centered cubic (fcc) lattice plane (111) parallel to the surface, a (2 20) reflection is favored for higher 
deposition pressures (A3), competing with the former. These diffractions correspond to a solid-solution of Pd-Ag with an fcc lattice. In this type of fcc structure, the $\{111\}$ family of crystallographic planes has the densest atomic packing. Moreover, these crystallographic planes possess the lowest surface energy, thus providing a stronger adatom mobility and tighter microstructure, as seen in Fig. 4a for the A1 membrane. It has been reported by other authors that atomic hydrogen permeates more efficiently across palladium fcc (111) planes [6], hence it is expected that process conditions leading to larger (111) facets will enhance the hydrogen selectivity. From the same XRD patterns, it is noted that the lattice parameters for membrane A3 are slightly strained compared to A1, which is attributed to the higher Ag content in this sample that inevitably leads to a higher distortion in the crystal lattice due to the larger Ag ionic radius ( $115 \mathrm{pm}$ and $86 \mathrm{pm}$ for $\mathrm{Ag}^{+}$and $\mathrm{Pd}^{2+}$, respectively).

Following this initial optimization procedure, a second series of membranes was produced, B1 and B2, with the aforementioned lower sputtering pressure of $0.43 \mathrm{~Pa}$, albeit reducing the $\mathrm{Pd}$ target current density to $8.1 \mathrm{~mA} / \mathrm{cm}^{2}$, as seen in Table 1, in order to enhance the Ag content so that hydrogen embrittlement in the film is minimized [3]. This resulted in slightly thinner membranes and an increased silver content of $23.1 \mathrm{wt}$ \% for membrane B2, which was deposited for $30 \mathrm{~min}$ (A series was $20 \mathrm{~min}$ ). As it will be shown later in this manuscript, this provided optimized conditions for these membranes. In Fig. 6a and b it is observed the surface and cross-section of membranes B1 and B2, respectively. Membrane B1 has a smoother and a more homogeneous surface, with less defects, compared to A1 (Fig. 4a). Due to the increase in silver content (by reducing the $\mathrm{Pd}$ target density during deposition), in B1 the stresses are best accommodated in the thin film microstructure and no visible cracks are observed. The layered cross-sectional view of membrane B2 in Fig. 6b enables the distinctive analysis of the interfacial adhesion layer of $\mathrm{YSZ}-\mathrm{g}-\mathrm{Al} 2 \mathrm{O} 3$, and the Pd-Ag film with its characteristic columnar microstructure. The film surface waviness replicates closely that of the underlying support. Regarding the solgel deposited YSZ-g-Al2O3 interfacial layer, the bottom part is distinct from its top amorphized surface; this separation was induced by the drying and calcination procedure described in the experimental section.

For the final gas-permeation measurements a new series of membranes ( $\mathrm{C} 1$ and C2) were deposited and compared to the B1, as reported in Table 1. For the C1 and C2 membranes, the Pd target density was further reduced to $7.6 \mathrm{~mA} / \mathrm{cm}^{2}$, yielding a maximum silver content of rv25 wt.\%. Furthermore, the deposition pressure was also reduced to $0.37 \mathrm{~Pa}$, with the objective to reduce $\mathrm{N} 2$ permeance and enhance membrane selectivity toward hydrogen. As it can be seen from Fig. 7 and Table 1, by varying the N2 pressure difference in the range of $100-500 \mathrm{kPa}$, the $C$ series membranes did not result in lower permeance values, when 
compared to membrane B1. The lowest permeance toward N2 was recorded for B1 sample $\left(0.076 \times 10^{-6} \mathrm{~mol} \mathrm{~m}^{-2} \mathrm{~s}^{-1} \mathrm{~Pa}^{-1}\right)$, in contrast to $>0.21^{-6}$ mol m-2 $\mathrm{s}^{-1} \mathrm{~Pa}^{-1}$ for $\mathrm{B} 2$ and following series $\mathrm{C} 1$ and $\mathrm{C} 2$ membranes, despite B1 being the thinnest membrane produced. It should be noted that, in fact, this nitrogen permeance results from Knudson flow through fissures in the thin film membrane microstructure.

From the previous results, membrane B1 was chosen for the $\mathrm{H} 2$ permeation tests, since it exhibited the lowest permeance toward nitrogen. Fig. 8 shows the membrane permeance to $\mathrm{H} 2$ and $\mathrm{N} 2$ as a function of the pressure difference through the membrane for sample B1. The permeate flow rate of hydrogen is larger than that of nitrogen, which yields evidence that this Pd-Ag membrane has a selectivity toward $\mathrm{H} 2$ with respect to $\mathrm{N} 2$. Hydrogen can permeate by sorptiondiffusion of atomic hydrogen, which is intended; and by viscous flow and Knudsen diffusion in the presence of leaks/ cracks or activated diffusion when micropores are present. Nitrogen only permeates when pores are present being then a probe for membrane defects. Since the pores and defects in the coating microstructure are of the order of the nm-size, viscous flow may be ruled out. Hence, the nitrogen permeance through the coating microstructure results from Knudsen diffusion through the above-mentioned defects.

It was found that for a pressure difference of $300 \mathrm{kPa}$ the $\mathrm{H} 2-\mathrm{flux}$ is $0.21 \mathrm{~mol} \mathrm{~m}^{-}$ $2 \mathrm{~s}^{-1}$, which corresponds to a permeance of $0.71 \times 10^{-6} \mathrm{~mol} \mathrm{~m}^{-2} \mathrm{~s}^{-1}$ $\mathrm{Pa}^{-1}$ (cf. Fig. 8), yielding an ideal selectivity of $\alpha\left(\mathrm{H}_{2} / \mathrm{N}_{2}\right)=10$. This value is only moderate indicating the presence of small pores in the membrane; hence, further optimization must be carried out.

By comparing the present results with those found in the literature for the same $\mathrm{Pd}-\mathrm{Ag}$ coating system deposited by magnetron sputtering, one concludes that the selectivity of the thin film Pd-Ag membranes of the present work is still rather low when compared to another optimized system found in the literature [20], where a selectivity greater than one order is obtained for $\mathrm{H} 2$ permeance experiments produced at high temperatures $\left(T=300{ }^{\circ} \mathrm{C}\right)$, albeit having a similar $\mathrm{H} 2$ flux across the membrane. It should be noted that the membranes in [21] are stand-alone films bonded to the final support, unlike the present work, where the films are sputtered directly onto the support and thus much thinner, reducing their cost. Conversely, in comparison to the Pd films deposited on a porous nickel support by magnetron sputtering [22] and $\mathrm{Pd}-\mathrm{Ag}$ on porous stainless steel by electroless plating [23], the present results are generally improved. As above-mentioned, it is important to note that all of our permeance experiments ( $\mathrm{N}_{2}$ and $\mathrm{H}_{2}$ ) were carried at room temperature, whereas for all referenced results the experiments were performed in the range of $300-500^{\circ} \mathrm{C}$. Furthermore, magnetron sputtering provides a cleaner and more 
environmentally-friendly technology, when comparing to other deposition techniques, such as chemical vapor deposition and electroless plating, providing thinner coatings and hence reduction in material cost.

The underachievement of the present work regarding $\mathrm{H}_{2}$ selectivity is possibly related to excessive intrinsic residual stress in the film, defects in the coating microstructure or in the porous ceramic supports. Additionally, since all permeance tests were performed at RT, hydrogen embrittlement is greater in these conditions, thus possibly resulting in the observed tensile cracks and other microstructural defects in the Pd-Ag membrane films that will in turn decrease membrane performance and selectivity toward hydrogen. Further work will be carried out to modify the coating microstructure in order to obtain higher selectivity toward hydrogen, in particular at higher temperatures, where a reactor is currently being idealized for this purpose.

\section{Conclusions}

A columnar structure in the $\mathrm{Pd}-\mathrm{Ag}$ thin film membranes is important for obtaining a good selectivity toward hydrogen and hindrance to other gases permeation. Furthermore, the columnar microstructure has to be compact to avoid the formation of voids between columns, which inevitably yields leakages. The use of low working pressures and heating to $200{ }^{\circ} \mathrm{C}$ during $\mathrm{Pd}$ and Ag co-sputtering deposition further enhances the aforementioned selectivity.

The use of a homogeneous and smooth YSZ-g-Al2O3 coat layer over the $a-$ alumina tubular support promoted a good adhesion of sputtered $\mathrm{Pd}-\mathrm{Ag}$ thin film. It was also found that a good surface finish, with low roughness and free of defects, is necessary to induce a uniform growth of the metallic membrane with an optimum structural organization and surface morphology.

A preferred orientation along [111] associated to a face-centered cubic cell is favored at low working pressure, yielding a stronger adatom mobility during growth, which will in turn enhance atomic hydrogen permeation across the membrane structure. In future work, it will be necessary to perform additional permeance tests at higher temperatures in order to activate the mechanism of sorption-diffusion that will increase membrane selectivity toward hydrogen and also H2 flux. This step is crucial, since hydrogen embrittlement at RT is perhaps the main reason for the relatively lower selectivity encountered for the membranes of the present work, when comparing to others from the literature. Furthermore, the porous ceramic supports should be monitored to avoid micro cracks that may significantly induce a replication of the defect structure in the thin film. 


\section{Acknowledgements}

Patricia Pérez is grateful to Fundação para a Ciência e a Tecnologia (FCT) for the doctoral grant (reference: SFRH/BD/ 73673/2010). The authors also acknowledge financing from FCT through the project PTDC/EQUERQ/098730/2008 and COMPETE scientific program.

\section{Appendix A. Supplementary data}

Supplementary data associated with this article can be found, in the online version, at http://dx.doi.org/10.1016/j.materres- bull.2014.10.055.

\section{References}

1. D. Mendes, A. Mendes, L.M. Madeira, A. Iulianelli, J.M. Sousa, A. Basile, Asia Pac. J. Chem. Eng. 5 (2010) 111-137.

2. J.D. Holladay, J. Hu, D.L. King, Y. Wang, Catal. Today 139 (2009) 244-260.

3. H.D. Tong, a. H.J. Vanden Berg, J.G.E. Gardeniers, H.V. Jansen, F.C. Gielens, M.C. Elwenspoek, Thin Solid Films 479 (2005) 89-94.

4. S. Yun, S. Ted Oyama, J. Membr. Sci. 375 (2011) 28-45.

5. J. Okazaki, D. Tanaka, M. Tanco, Y. Wakui, F. Mizukami, T. Suzuki, J. Membr. Sci. 282 (2006) 370-374.

6. Basile, A. Iulianelli, T. Longo, S. Liguori, M.D. Falco, Membrane Reactors for Hydrogen Production Processes, Springer, London, 2011.

7. W. M. Tucho, Self-supported, thin Pd/Ag membranes for Hydrogen separation, Norwegian University of Science and Technology, PhD Thesis, 2009.

8. N. Lopez, Z. Łodziana, F. Illas, M. Salmeron, Phys. Rev. Lett. 93 (2004) 1-4.

9. W. Mekonnen, B. Arstad, H. Klette, J.C. Walmsley, R. Bredesen, H. Venvik, R. Holmestad, J. Membr. Sci. 310 (2008) 337-348.

10. [10] B.A. McCool, Y.S. Lin, J. Mater. Sci. 6 (2001) 3221-3227.

11. M.L. Bosko, J.B. Miller, E.A. Lombardo, A.J. Gellman, L.M. Cornaglia, J. Membr. Sci. 369 (2011) 267-276.

12. B. McCool, G. Xomeritakis, Y. Lin, J. Membr. Sci. 161 (1999) 67-76.

13. D.A. Pacheco Tanaka, M.A. Llosa Tanco, J. Okazaki, Y. Wakui, F. Mizukami, T.M. Suzuki, J. Membr. Sci. 320 (2008) 436-441.

14. G.Y. Meng, L. Huang, M. Pan, C.S. Chen, D.K. Peng, Mater. Res. Bull. 32 (1997) 385-395.

15. Y. Wu, L. Zhang, G. Li, C. Liang, X. Huang, Y. Zhang, G. Song, J. Jia, Z. Chen, Mater. Res. Bull. 36 (2001) 253-263.

16. Mejdell, T.A. Peters, M. Stange, H.J. Venvik, R. Bredesen, J. Taiwan Inst. Chem. E 40 (2009) 253-259. 
17. H. Zhao, G. Xiong, N. Stroh, H. Brunner, Sci. China Ser. B 42 (1999) 581-588.

18. R. Mallada, M. Menéndez, Membrane Science and Technology Series, Inorganic Membranes: Synthesis, Characterization and Applications, Ed. U. Zaragoza, 2008.

19. D.M. Mattox, Handbook of Physical Vapor Deposition (PVD) Processing Film Formation, Adhesion, Surface Preparation and Contamination Control, Noyes Publications, Westwood, New Jersey, 1998.

20. [20] B.K.R. Nair, M.P. Harold, J. Membr. Sci. 290 (2007) 182-195.

21. A.L. Mejdell, H. Klette, A. Ramachandran, A. Borg, R. Bredesen, J. Membr. Sci. 307 (2008) 96-104.

22. S.-K. Ryi, J.-S. Park, S.-H. Kim, S.-H. Cho, J.-S. Park, D.-W. Kim, J. Membr. Sci. 279 (2006) 439-445.

23. J. Tong, R. Shirai, Y. Kashima, Y. Matsumura, J. Membr. Sci. 260 (2005) 84-89

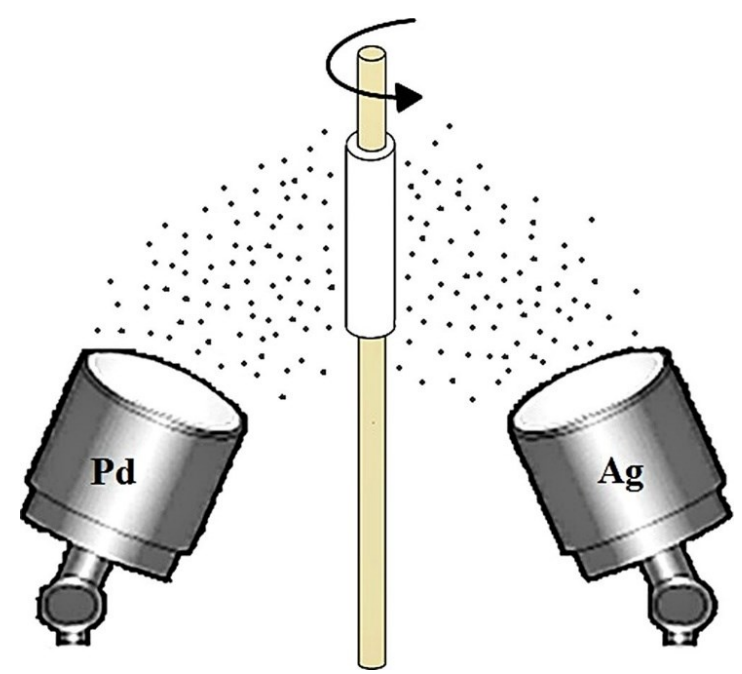

Fig. 1. Schematic of the co-sputtering procedure associated with the Pd-Ag thin film deposition on the ceramic membrane support.

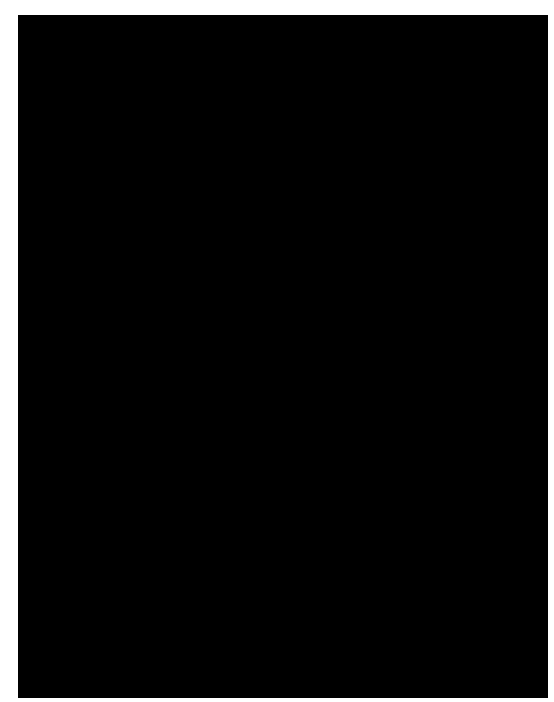

Fig. 2. Schematic of the gas-permeation test procedures with the $\mathrm{Pd}-\mathrm{Ag}$ membranes. 


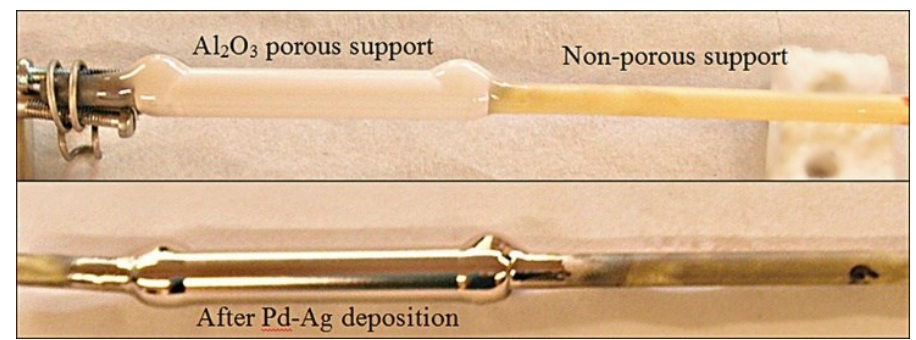

Fig. 3. Images of the ceramic support, capped with the sol-gel-deposited YSZ- $\gamma-\mathrm{Al} 2 \mathrm{O} 3$ layer (top) and after the deposition of the Pd-Ag thin film (bottom). 

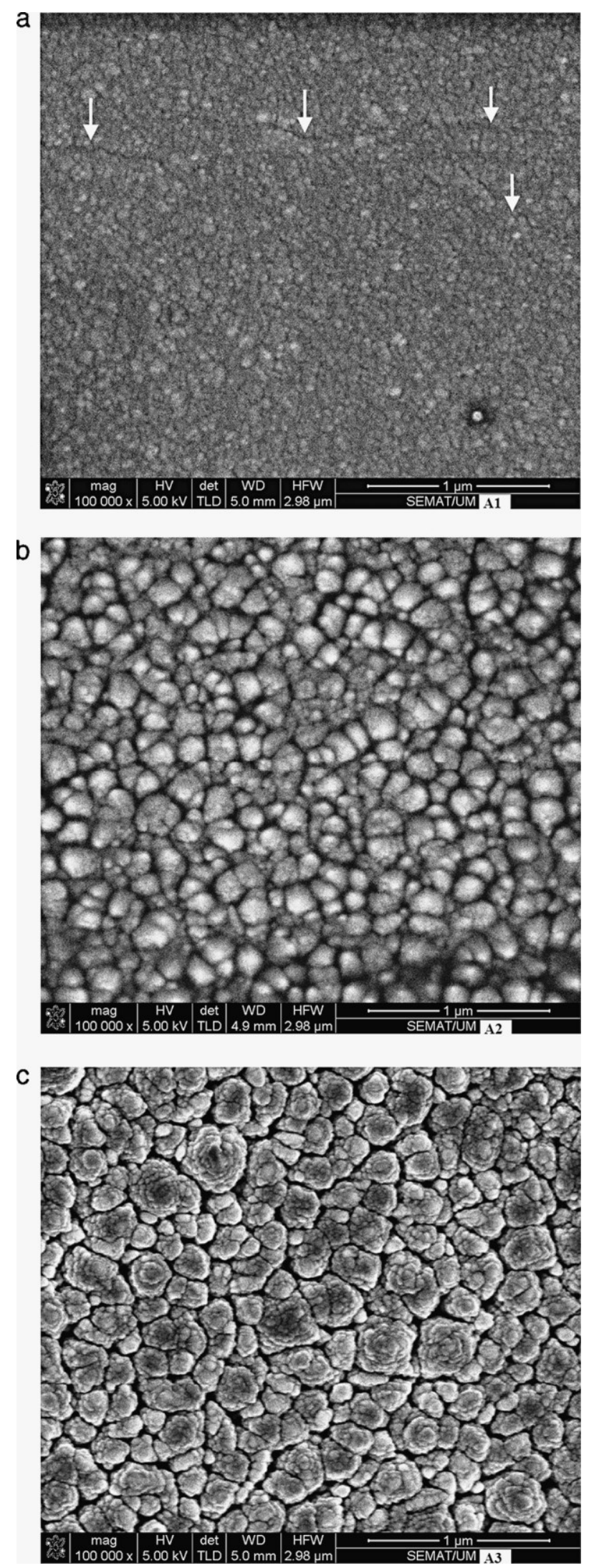

Fig. 4. SEM micrographs of the surface topography of the Pd-Ag thin film onto the porous support, for 3 different samples: (a) A1 (tensile cracks indicated by arrows); (b) A2 and (c) A3. 


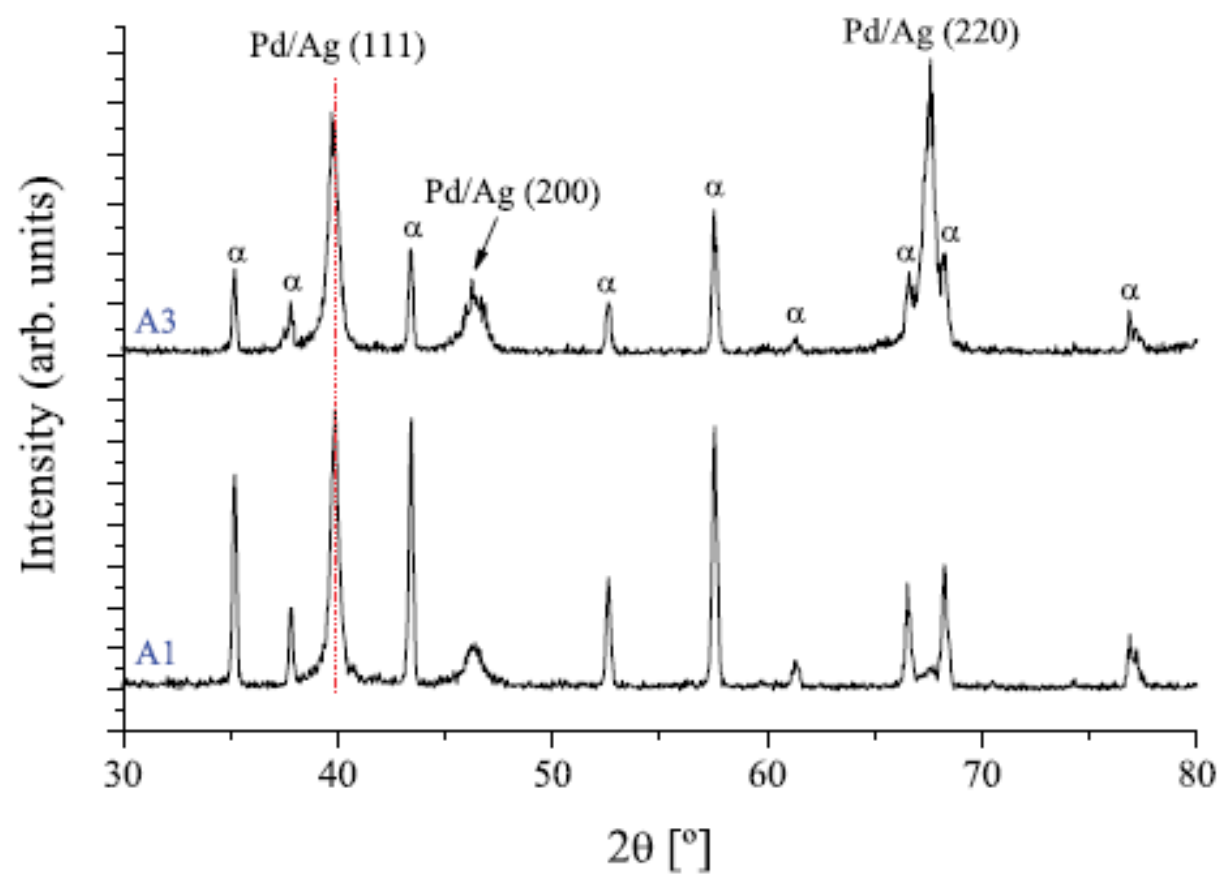

Fig. 5. X-ray diffraction patterns for samples A1 and A3; $a$ denotes diffraction peaks from the $a$-alumina porous support. 

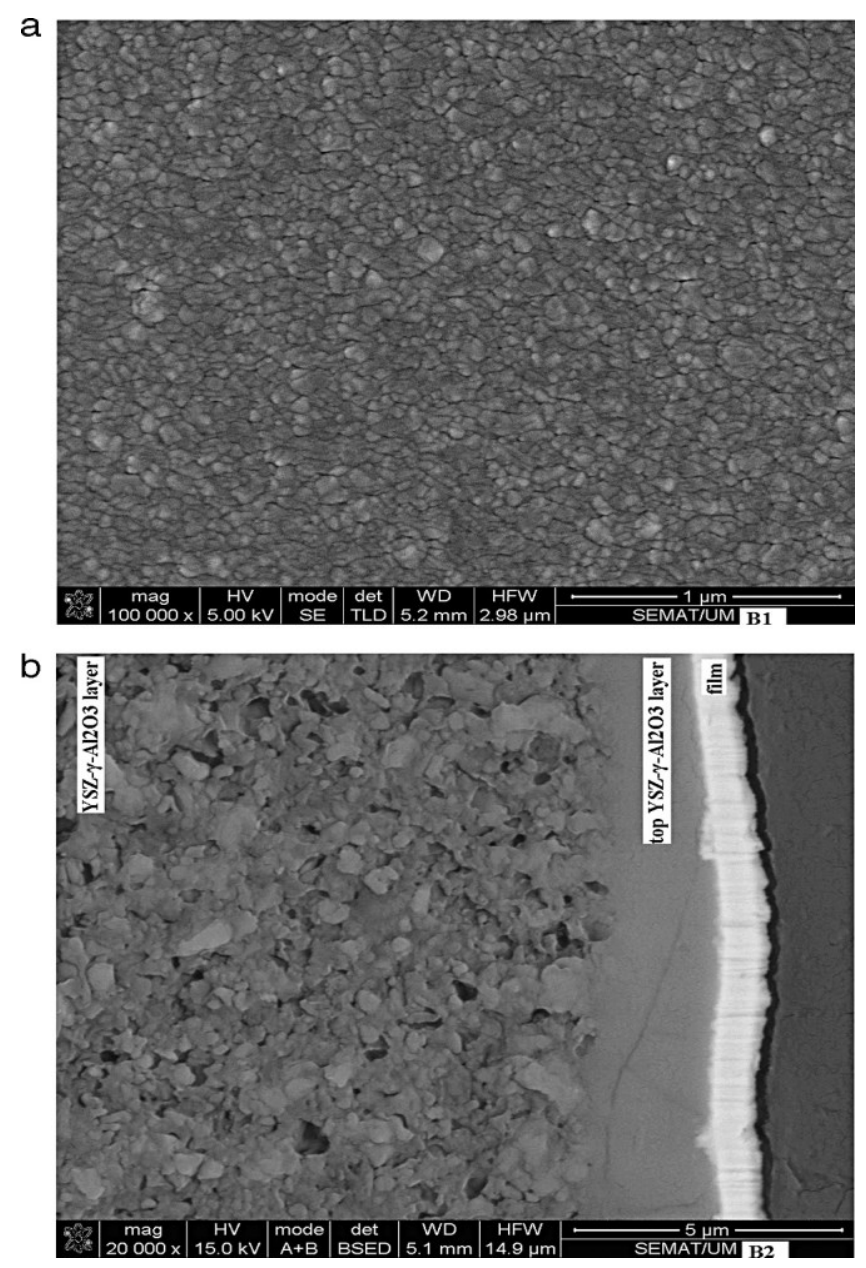

Fig. 6. SEM micrographs relative to sample B1 and B2, showing the (a) surface topography and (b) cross-section, respectively.

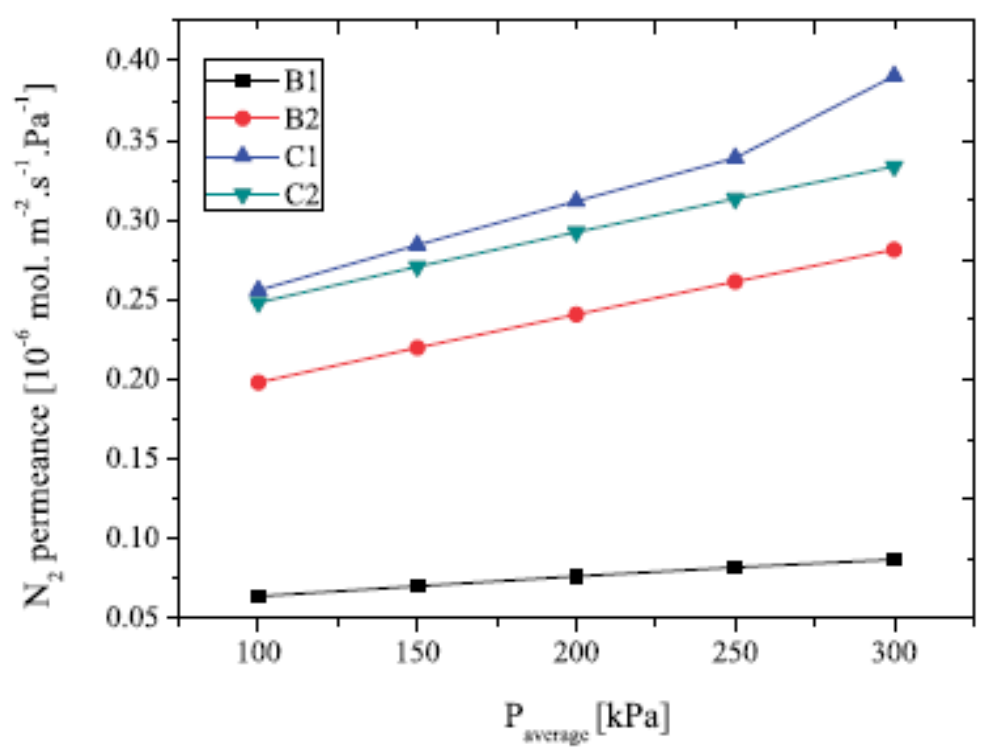

Fig. 7. Membrane permeance toward nitrogen as a function of the pressure difference through the membranes B1, B2, C1 and C2; permeate pressure is the ambient pressure. 


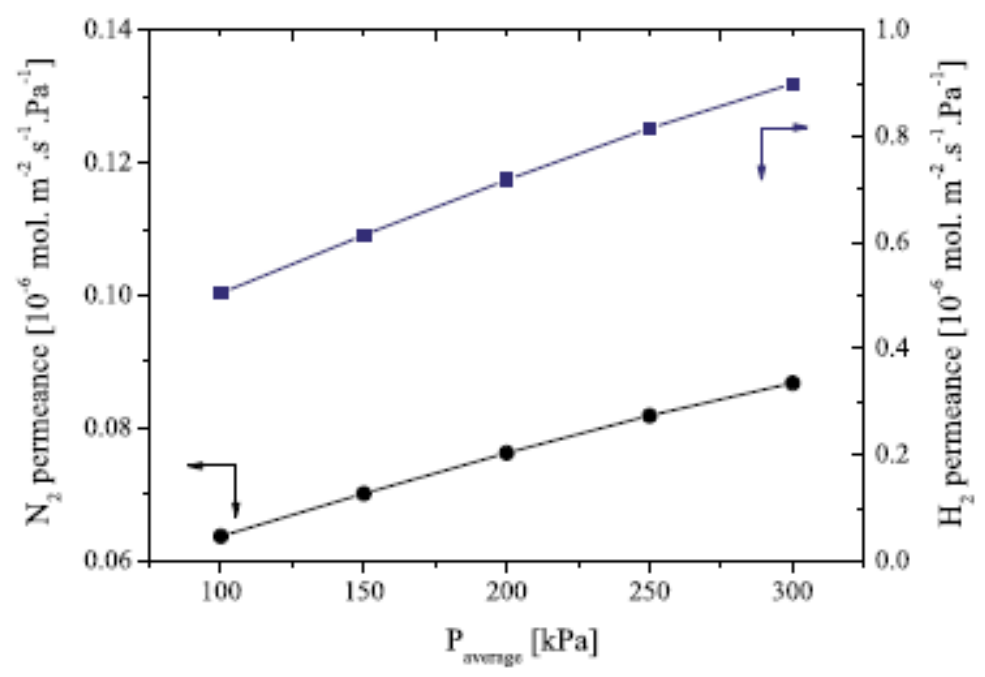

Fig. 8. Membrane permeance toward nitrogen and hydrogen as a function of pressure difference through the membrane B1; permeate pressure is the ambient pressure. 
Table 1

Selected samples and respective specific PVD process parameters for the deposition of the Pd-Ag thin films, including thickness and wt.\% of Ag, and corresponding nitrogen permeance.

\begin{tabular}{llllllll}
\hline Sample & Dep. time $[\mathrm{min}]$ & Thick. $[\mu \mathrm{m}]$ & $\mathrm{JPd} / \mathrm{Agg}\left[\mathrm{mA} / \mathrm{cm}^{2}\right]$ & wt.\% $(\mathrm{Ag})$ & Argon $[\mathrm{sccm}]$ & Dep. pressure $[\mathrm{Pa}]$ & $\mathrm{N}_{2}$ permeance $\left[\times 10^{-6} \mathrm{~mol} \mathrm{~m}^{-2} \mathrm{~s}^{-1} \mathrm{~Pa}{ }^{-1}\right] \mathrm{at} 200 \mathrm{kPa}^{-}$ \\
\hline A1 & 20 & 0.73 & $9.2 / 1.6$ & 14.8 & 50 & 0.43 & 0.91 \\
A2 & 20 & 0.97 & & 17.5 & & 1 & n.a. \\
A3 & 20 & 1.41 & & 19.8 & & 3 & n.a. \\
B1 & 20 & 0.71 & $8.1 / 1.6$ & 18.1 & & 0.43 & 0.076 \\
B2 & 30 & 1.06 & & 23.1 & & 0.43 & 0.241 \\
C1 & 25 & 0.91 & $7.6 / 1.6$ & 25.3 & 35 & 0.37 & 0.312 \\
C2 & 30 & 1.18 & & 25.1 & & 0.37 & 0.292 \\
\hline
\end{tabular}

